\title{
È stata richiesta una consulenza su consulenziale
}

Anna M. Thornton

PUBBLICATO: 07 APRILE 2020

\section{Quesito:}

Diversi lettori ci chiedono se sia corretto l'aggettivo consulenziale che non hanno trovato nei dizionari da loro consultati.

\section{È stata richiesta una consulenza su consulenziale}

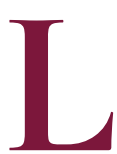

a risposta breve è: sì. Consulenziale è registrato nei repertori più aggiornati, quali il Supplemento 2004 al GDLI, dove è definito "che è proprio, che si riferisce alla prestazione professionale di un consulente" e datato al I992; analoga definizione e stessa data (probabilmente dipendente dal GDLI) si trova nel vol. II di Nuove parole italiane dell'uso, vol. VIII del GRADIT, pubblicato nel 2007; qui il termine è qualificato come di uso burocratico e commerciale.

La prima attestazione della voce consulenziale in italiano può essere retrodatata di qualche decennio, grazie alla ricerca del termine nel repertorio di Google Libri: nel 1956 consulenziale appare negli Acta medicinae legalis et socialis, nel seguente contesto: "Dopo anni di attività consulenziale esercitata in Milano, su incarichi del Tribunale e della Corte di Appello" (p. 595); nel ig65 riappare nella rivista Produttività: "quasi zoo aziende hanno spontaneamente manifestato la loro soddisfazione per l' attività consulenziale svolta dai tecnici del Centro" (p. 50).

La derivazione di aggettivi in -iale (invece del semplice -ale) da basi in -enza in italiano contemporaneo ha carattere sistematico, come illustrato in Anna M. Thornton, On Italian derivatives with antesuffixal glides, in G. Booij \& J. van Marle (a cura di), Yearbook of Morphology 1998, I999, pp. I03-I26. Per un parallelo si pensi a un'altra voce di attestazione relativamente recente, emergenziale (datata al I984 nel vol. I di Nuove parole dell'uso, vol. VII del GRADIT, 2003). Nel Nuovo De Mauro a fronte di oltre Ioo formazioni in -enziale si hanno tre sole voci in -enzale: provenzale (con il composto francoprovenzale), senz'altro modellato sulle forme di provenzale antico provensal e di francese provençal; influenzale (con i suoi prefissati: anti-influenzale, ecc.) e semenzale.

La presenza di / $\mathrm{j}$ / tra una base in -enza e il suffisso -ale si spiegherebbe facilmente se l'aggettivo fosse stato derivato già in latino, da una base in -entia. Tuttavia, non è questo il nostro caso.

Aggettivi in -entialis in latino sono rari e di formazione tarda: il più antico è forse sapientialis, attestato in Tertulliano (II-III secolo d.C.); qualche altra formazione (alla base di esistenziale, essenziale, pestilenziale, presenziale) si ha in latino tardo o medievale; ma molti degli aggettivi italiani in -enziale sono adattamenti di prestiti dal francese (per es. esponenziale; evenemenziale <événementiel, derivato diévénement 'avvenimento'; preferenziale; presidenziale) o dall'inglese (consequenziale, referenziale), i cui modelli già presentano una $i$ ortografica prima del suffisso corrispondente a -ale. Sulla base di questi modelli, anche la massima parte degli aggettivi di formazione italiana da nomi in -enza termina in -enziale, come il nostro consulenziale, nonostante non risalga a una forma latina in -entialis. D'altronde, la stessa voce consulentia è di attestazione tarda in latino: manca negli autori classici; il Thesaurus Linguae Latinae (TLL) ne offre alcune attestazioni in autori cristiani del IV-V secolo, quali Rufino e Agostino, nelle cui opere vale piuttosto "cura, consilium, providentia", e non ha 
certo il senso dell'italiano consulenza 'prestazione professionale di un consulente' (GRADIT e Zingarelli 2019), che pure lo continua formalmente.

La voce consulenza (datata I92 nel GRADIT e nello Zingarelli 2019) in italiano appare all'inizio del XX secolo in pubblicazioni burocratiche, come il Bollettino dell'Ufficio del lavoro pubblicato dal Ministero di Agricoltura, Industria e Commercio, vol. II, n. I, agosto I904, dove ha 5 occorrenze, la prima delle quali in una notizia sul "Congresso della Federazione tra Sindacati e Sodalizi di ferrovieri" del giugno r9o3, il quale "deliberò che la discussione del memoriale coi rappresentanti del Governo debba essere fatta col mezzo di due Commissioni: una per le trattative dirette, l'altra di consulenza e di controllo"; poco oltre tale commissione è chiamata "Commissione di consulenza" (p. I44); nello stesso numero del Bollettino si cita poi un ufficio "di consulenza legale e medica" (p. I45), e ancora altre due volte la locuzione "consulenza legale". Da questa voce è stato poi creato l'aggettivo consulenziale, come documentato sopra.

Sia consulenza che consulenziale non sembrano avere paralleli in inglese e in francese: voci quali *consulence, *consulential e *consulence, "consulentiel sono assenti rispettivamente nell'Oxford English Dictionary (OED) e nel Trésor de la Langue Française informatisé (TLFi). Allitaliano consulenza corrispondono in francese consultation, in inglese consultancy o legal advice. La percezione di "stranezza" dell'aggettivo consulenziale da parte dei nostri lettori è quindi comprensibile, dato che la voce, come anche la sua base consulenza, si trova un po' isolata nel panorama delle lingue europee moderne con cui siamo maggiormente in contatto. Tuttavia, l'aggettivo italiano consulenziale è perfettamente ben formato secondo le regole oggi vigenti nella nostra lingua.

\section{Cita come:}

Anna M. Thornton, È stata richiesta una consulenza su consulenziale , "Italiano digitale", 2019, XIII, 2020/2 (aprile-giugno)

DOI: $10.35948 / 2532-9006 / 2020.3288$

Copyright 2019 Accademia della Crusca

Pubblicato con licenza creative commons CC BY-NC-ND 